\title{
A Colour Analysis Tool for Product Appearance
}

\author{
Cheng-Min Tsai ${ }^{1}$ and Xue $\mathrm{Gao}^{2}$ \\ ${ }^{1}$ Department of Visual Arts and Design, Nanhua University, Chiayi, 622, Taiwan, R.O.C. \\ ${ }^{2}$ School of Art and Design, Zhengzhou Sias University, Xinzheng, Henan, 451150, China.
}

\begin{abstract}
This study proposes an image colour analysis tool that could help graphic/product designers to understand colour distribution and colour appearance of a series product. The analysis method is in accordance with the CIEL $a b$ uniform colour space created by the International Commission on Illumination (CIE) in 1976. Based on the analysis method used in a previous study of the oil paintings, 370 oil paintings were analysed. The results demonstrated that using simple colour difference formula could reveal the images' numerical colour distribution (Tsai, 2019). The focus of this study is twofold: to understand the colour appearance of product design and to check the rationality and reliability of the analysis tool. The analysis tool this study proposes could also be useful in providing the product designers with easy analysis and understanding of the colour distribution of images.
\end{abstract}

\section{Introduction}

Marketing strategies aim to determine the position of target consumers, including terms of age, sexual preference and popular trend in marketing. Product colour planning is quite an important issue in marketing strategies. How to analyse and understand the colour used in current products is one of the important training topics for graphic/product designers. Whether it is colour analysis of product design or colour training of the graphic design, a robust colour analysis method plays a critical role to accurately understand the colours used. The personal knowledge of the viewer tends to affect the reading of colour images. This is explained by how the human brain processing of more complex colour images or colour chips might be dependent on previous knowledge, cognition and perception, such as when a person has received a substantial colour distribution of an image. Solso (1996) states that the meaning and interpretation of art depend on the viewer's previous specialized knowledge of images/painting and related phenomena. Thus, a robust colour analysis tool could assist the designers to objectively know how colour distribution and how many colour used in the uniform colour space. Thus, the first question addressed by this study is how to know the principal colours used in the appearance of the product. The second question is how to effectively analyse and easily compare the colours on the appearance of the product for providing the product designers' evaluation. Different from the humans' visual system, the main goal of colour science analysis is to develop a numerical metric based on image quantitative analysis to assess colour images. Tsai (2019) points out that a lot of image research field focused on the colour metrics were concerned about the physical measurement of image assessment and does not correspond well with the visual assessment results. In recent years, numerous studies were conducted based on visual assessments by using psychophysical methods to analyse the image reproduction and its application on painting artworks (Tsai, 2019). But, the aforementioned methods were based on a global analysis of the entire image. Thus, this study proceeded from the idea that colour science could reasonably analyse the colour or colours used in product design. What sets this study apart from previous research undertakings is that it utilised an effective way of analysing colour images in product design. While it is easy to select a colour from an image, it is difficult to decide which colour could represent the single product's colour appearance amidst how colour appearance is affected by texture, lightness, near colour, and background colour. For example, Figure 1 shows the sample of the colour appearance of a famous kettle. If a product designer would like to determine which colour accounts for the beautiful appearance of the kettle, they would have to try to pick up the colour from the colour appearance of the kettle, taking note that metal material, lightness and shadow all affect product appearance. The different areas of a product represent a different colour gradient (see Figure 1). The main purpose of this study is to develop a useful analytical tool to facilitate understanding of an image's colour distribution by providing the product designer's reference. 


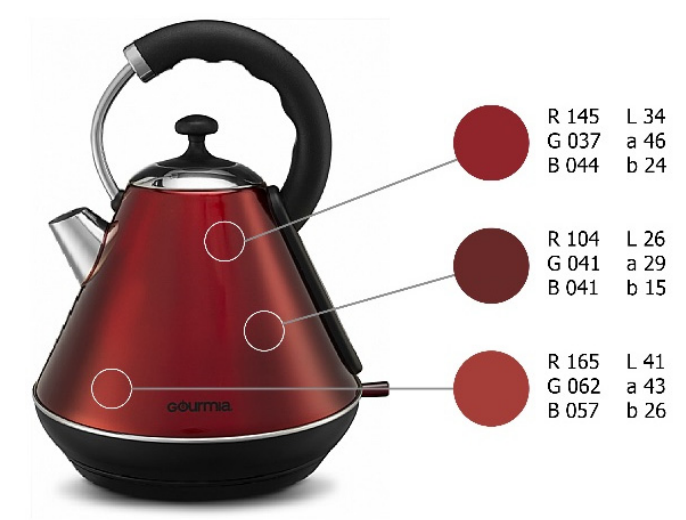

Fig. 1 the sample of the colour appearance of a kettle. (Gourmia, 2020) ${ }^{1}$

\section{Materials and Method}

Three images of monotone products have been analysed in this study: an image of three differently coloured kettles, an image of four differently coloured mobile phones and another kettle image, this time, four differently coloured kettles (see Figure 2). Each digitalised image included $\mathrm{R}, \mathrm{G}$ and $\mathrm{B}$ colour channels. The range of value for each colour channel is from integer 0 to 255 in each pixel of an image. Because unlike the numerical matrix of the $\operatorname{CIE} L^{*} a^{*} b^{*}$ colour value, the RGB values could not be easily analysed using basic arithmetic calculations, this study transferred the RGB to the $\operatorname{CIE} L^{*} a^{*} b^{*}$ value in each pixel of an image.

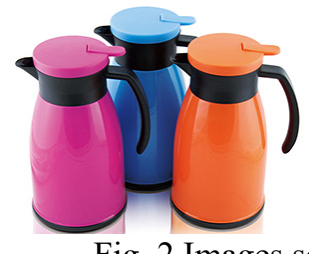

Fig. 2 Images set for analysis of

\subsection{CIELAB colour space}

In 1976, the International Commission on Illumination (CIE) defined the CIELab colour space, which was created to serve as a device-independent function to be used as a reference. Equations 1-4 were used to describe the tristimulus values normalized to the reference white. The CIELab colour space also defines the three dimensions, including $X, Y$, and $Z$, which are the tristimulus values of the stimulus, and $X_{n}, Y_{n}$, and $Z_{n}$, which are the tristimulus values of the reference white point (CIE, 1986; Fairchild, 2005). The CIELab colour
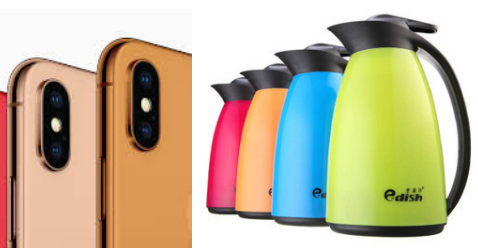

space sets up the colour as three dimensions of the CIE $L^{*}$ for lightness from zero (black) to one hundred (white) and the CIE $a^{*}$ and $\mathrm{CIE} b^{*}$ for the hue and chroma. The CIELab colour space was also designed as a "perceptually uniform or uniform colour space," which means that "the same amount of numerical colour value change corresponds to roughly the same amount of visually colour perceived change" (CIE, 1986; Brainard, 2003). This study calculates the numerical colour volume of an image by using CIELab colour space (Tsai, 2019).

$$
L^{*}=116 \sqrt[3]{\frac{Y}{Y n}}-16 \quad a^{*}=500\left(\sqrt[3]{\frac{X}{X n}}-\sqrt[3]{\frac{Y}{Y n}}\right) \quad b^{*}=200\left(\sqrt[3]{\frac{Y}{Y n}}-\sqrt[3]{\frac{Z}{Z n}}\right)
$$

When the $X / X_{n}, Y / Y_{n}$, and $Z / Z_{n}$ are greater than 0.008856 .

$$
\begin{aligned}
& L^{*}=903.3\left(\frac{Y}{Y n}\right) \\
& a^{*}=500\left\{\left[7.87\left(\frac{X}{X n}\right)+\frac{16}{116}\right]-\left[7.87\left(\frac{Y}{Y n}\right)+\frac{16}{116}\right]\right\} b^{*}=200\left\{\left[7.87\left(\frac{Y}{Y n}\right)+\frac{16}{116}\right]-\left[7.87\left(\frac{Z}{Z n}\right)+\frac{16}{116}\right]\right\}
\end{aligned}
$$

$\mathrm{W}^{1}$ hen the $X / X_{n}, Y / Y_{n}$, and $Z / Z_{n}$ are equal to or less than 0.008856 .

\footnotetext{
${ }^{1}$ The famous kettle design by Gourmia company. The copyright of the image belongs to the Gourmia company. (please see https://www.gourmia.com/)

${ }^{2}$ The copyright of the mobile phone's image belongs to the apple company (please see https://www.apple.com/); The copyright of the left side kettle's image belongs to the stationeryinfo.com (please see http://stationeryinfo.com/); The copyright of the right side kettle's image belongs to the Guangzhou Sure Up Commodity Limited Company. (please see https://sure-up.com/)
} 


\subsection{Colour analysis of the CIELAB}

A serial workflow used to obtain the RGB value of each pixel in the source image and transfer the colour value of each pixel to $\operatorname{CIEL} L^{*} a^{*} b^{*}$. Each pixel of an image started as sRGB data that is standardized from the RGB value. Each pixel is then transformed into the CIEXYZ colour space and then $L^{*}, a^{*}$, and $b^{*}$ values (see Eqs. $1-2$ ). The output of the workflow was to transfer the colour value of $\mathrm{CIE} X Y Z$ to $\mathrm{CIE} L^{*} a^{*} b^{*}$ (see Figure 3). Each $L^{*}, a^{*}$, and $b^{*}$ value started as sRGB data that is standardized from the RGB value in the workflow.

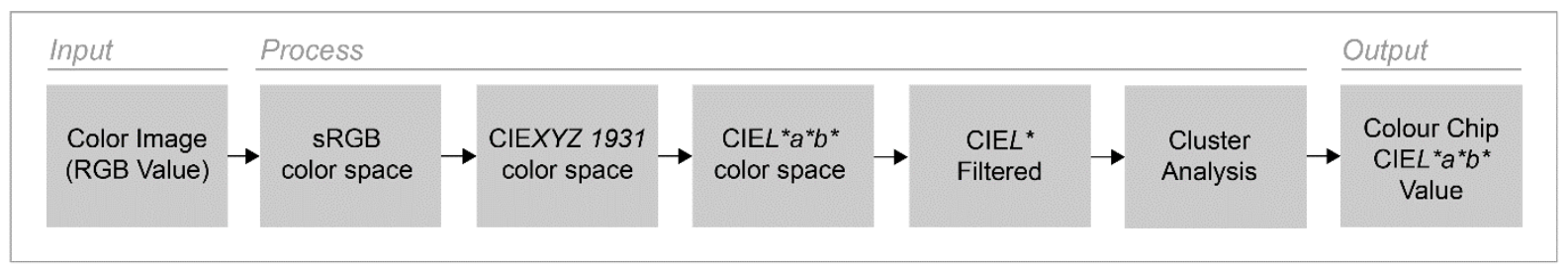

Fig. 3 Workflow to calculate the colour image (from RGB value to CIE $L^{*} a^{*} b^{*}$ )

\subsection{Colour analysis tool}

This study sought to develop an analysis tool and method that could easily analyse and understand the colour distribution of images. Visual Basic programming was used to develop the analysis tool's interface and function, including image file input, colour analysis, colour space represents and so on. The colour analysis was performed according to the workflow process for calculating the colour image from RGB value to $\operatorname{CIE} L^{*} a^{*} b^{*}$ (See Fig. 3). The process began from the inputted colour images' RGB value towards the $\mathrm{sRGB}$ value, then transferred to
CIEXYZ colour space and CIELab colour space. The calculation of the $\mathrm{CIE} L^{*} a^{*} b^{*}$ values was used to determine the colour distribution of an image. All the steps were according to the process used in the pilot study (Tsai, 2019). All the images would be input to the analysis tool used to obtain the RGB value of each pixel in the source image and to transfer the colour value of each pixel to $\mathrm{CIE} L^{*} a^{*} b$ values. Each pixel of an image would be represented as CIELab colour space (see Fig. 4). The distribution map shows the two axes with CIE $a^{*}$ and $\mathrm{CIE} b^{*}$ values.

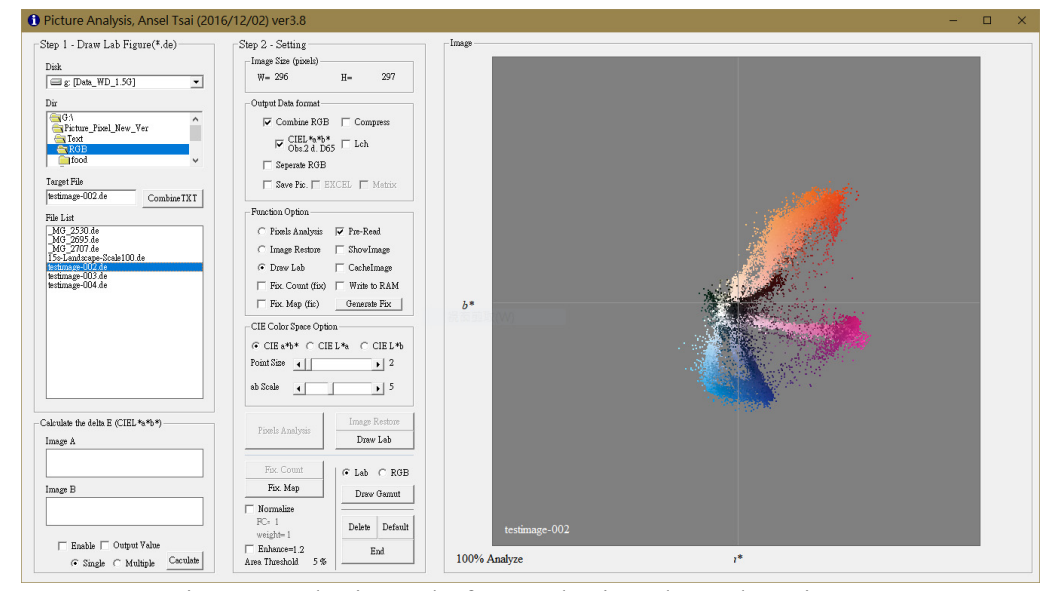

Fig. 4 Analysis tools for analyzing the colour image.

\subsection{The cluster analysis}

The K-means clustering technique was used for analysis in this study. The main purpose of cluster analysis is to group similar data in the three dimensions. K-means clustering aims to partition $n$ observations into $K$ clusters in which each observation belongs to the cluster with the nearest mean, serving as a prototype of the cluster. As an example in this study, the colour value of each pixel $(n)$ was set to three or four clusters $(K)$ in the CIELab colour space. The number of $K$ values was based on the number of primary colours in the image. As clusters include groups with small distances between observations, the centre of the group represented the small distances to each cluster member. Thus, the centre point of the group also represented the main colour with $\operatorname{CIE} L^{*} a^{*} b^{*}$ value.
As Figure 5 shows, three main colour groupings were derived from a lot of observation value $(n)$.

\section{Data Analysis}

It is clear that the three images' colour distribution may discriminate the colour group. The left figure shows three main colours, the middle image depicts four main colours, and the image showing three to four main colours are seen on the right figure (see Figure 5). Table 1 shows the results of the cluster analysis that the $\operatorname{CIE} L^{*} a^{*} b^{*}$ value corresponds to in the centre of each colour group. The right side shows the colour chip of each central colour representation by using $\operatorname{CIE} L^{*} a^{*} b^{*}$ value. To reduce the outliner data influence in the result of this study, the lightness data below the Q1 and over the Q3 have been filtered using the $\mathrm{CIE} L^{*}$ value. Conversely, the lightness 


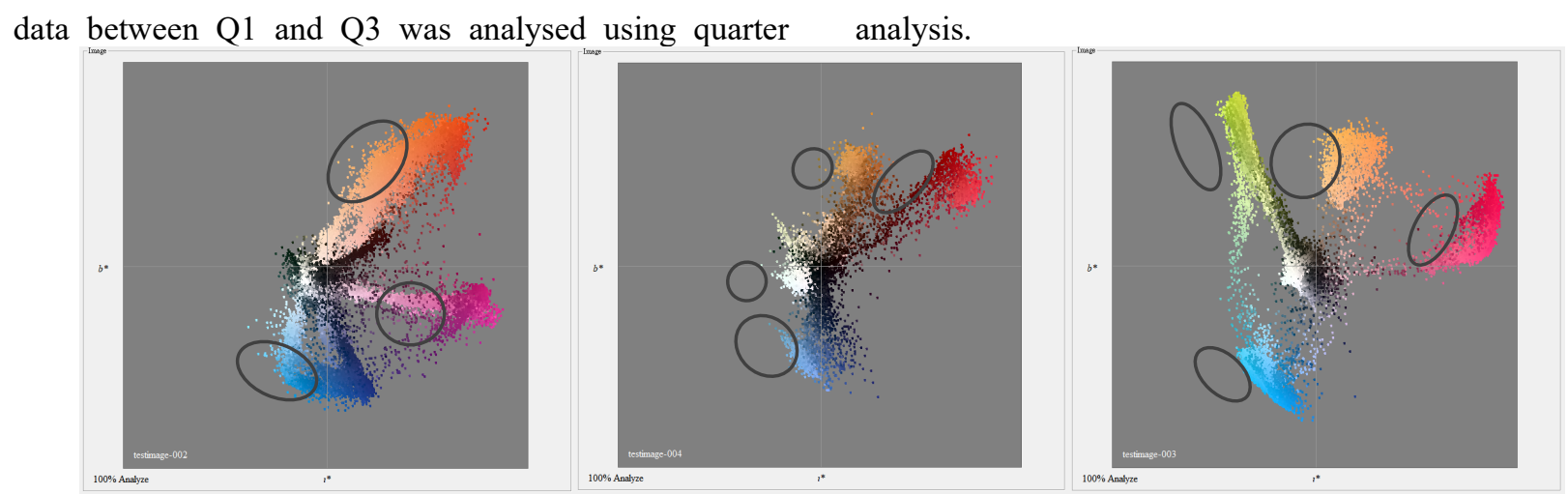

Fig. 5 the colour distribution of the colour images in the CIELab colour space. (the $\mathrm{x}$ axis is CIE $a^{*}$; the $\mathrm{y}$ axis is CIE $b^{*}$ )

Table 1. Results of cluster analysis.

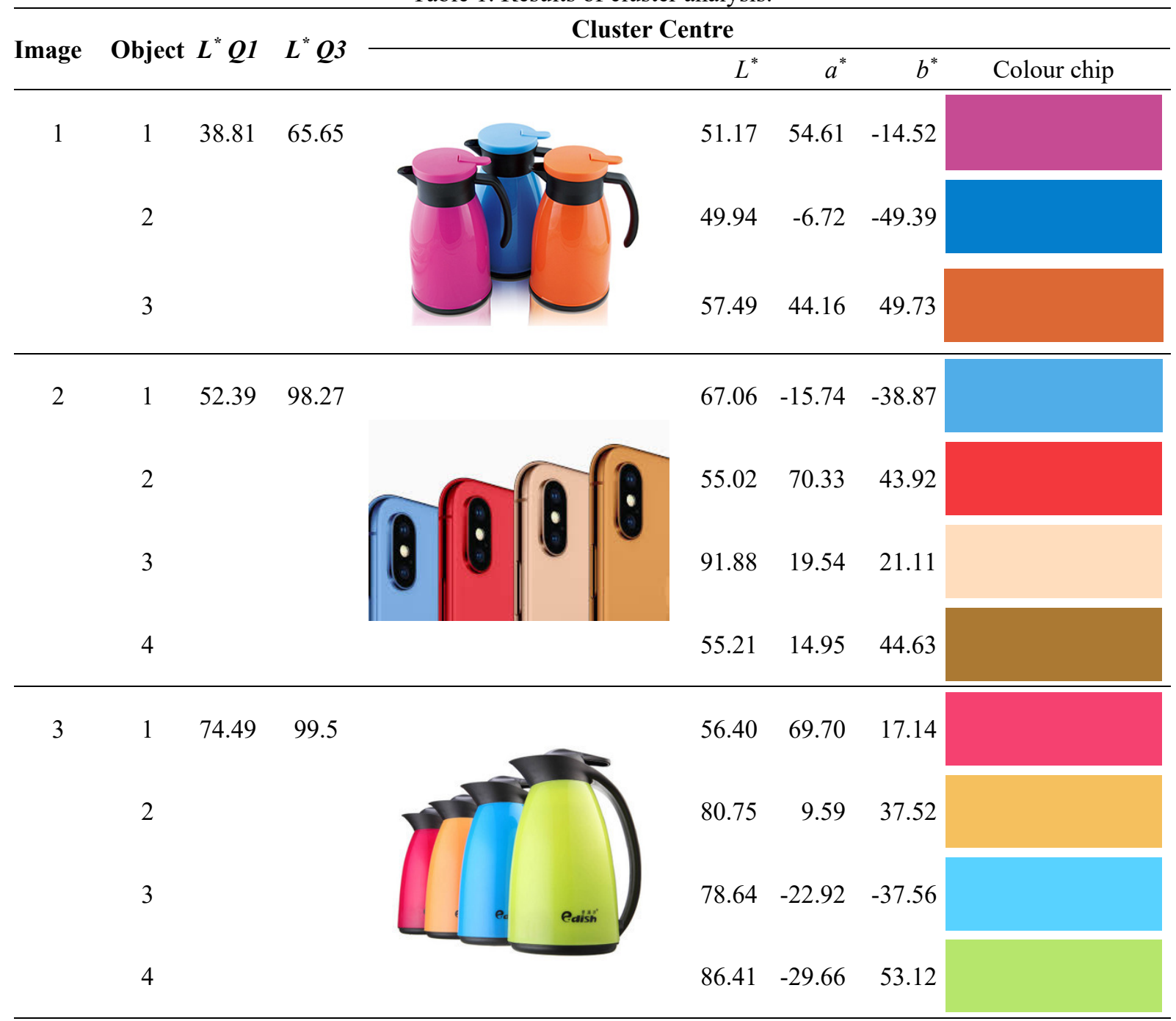




\section{$4 \quad$ Results and Discussion}

This study focused on understanding the colour appearance of product design and checking the rationality and reliability of the analysis tool proposed herein. The results showed that colour chips could be more easily understood by using the analysis method proposed in this study. Each colour chip could represent an image in CIE $L^{*} a^{*} b^{*}$ values. Different and superior in certain ways from the human visual system, the analysis tool used in this study could provide product designers with a means of easy analysis and objectively understanding of the colour distribution of images. According to the question and purpose addressed by this study, the analysis tool could effectively be used by product designers to quickly ascertain the principal colours used in the colour appearance of a product. The cluster centre could be easily represented as a primary colour very similar to the colour appearance of the product image. An application of CIE $L^{*} a^{*} b^{*}$ values could be easier to apply to commercial graphic design software; for example, the colour palette of Adobe Photoshop or Illustrator. Additionally, this study suggests practical applications for CIELCh values in the future; because of the $C$ value is according to the value of Chroma while the $h$ value represented the mean hue position. It might be another nice colour space to provide the designer to understand the colour distribution.

\section{Acknowledge}

Special thanks to the product's company who provided the images to analyse the colour appearance of an image in this study. All the images' copyright belongs to the company that holds the product's right. All the research data (e.g. colour value) only provide an academic issue, instead of use to a commercial purpose. The results of all colour value could not represent the colour appearance of the original product. All the images only used to test the function of the analysis tool in this study.

\section{References}

1. Tsai, C.M., Guan, S. S. (2005) Identifying regions of interest in reading an image, Displays, 39: 33-41.

2. Fairchild, M.D. (2005) Colour Appearance Models, The John Wiley \& Sons.

3. CIE (1986) Colourimetry, Commission Internationale de l'Eclairage (CIE) Publication, 15.2, Vienna.

4. Brainard, D.H. (2003) Colour appearance and colour difference specification. In Elsevier Science (Eds.), The Science of Colour (2nd Edition) (pp. 191-216). Amsterdam, Netherlands: Elsevier Science.

5. Solso, R.L. (1996) Cognition and the Visual Arts, A Bradford Book, Cambridge, Massachusetts, London: The MIT Press. pp. 101-127.
6. Tsai, C.M. (2019) Using CIELAB colour space in analyzing the colours of Van Gogh's paintings, in proceeding of 2019 International Academic Conference and Exhibition on Visual Arts and Design, Nanhua University, Chiayi, Taiwan. 